\title{
The descent of off-shell supersymmetry to the mass shell
}

\section{short review}

D. $\operatorname{Krotov}^{1,2,3, a}$

${ }^{1}$ Institute for Nuclear Research of the Russian Academy of Sciences, Moscow, Russia

2 Institute of Theoretical and Experimental Physics, Moscow, Russia

${ }^{3}$ Moscow State University, Department of Physics, Moscow, Russia

Received: 19 July 2007 / Revised version: 19 December 2007 /

Published online: 30 January 2008 - (c) Springer-Verlag / Società Italiana di Fisica 2008

\begin{abstract}
It is shown that classical actions for some "physically interesting" quantum field theories can be obtained as effective actions from the single "fundamental" theory of the Chern-Simons form. The physical degrees of freedom are encoded in the space of cohomologies of a certain differential operator. This observation suggests a different perspective on some of the supersymmetric properties of these effective theories. Namely, it is possible to construct a superfield formalism which allows to find off-shell SUSY actions for the on-shell supersymmetric theories, where conventional superfield formalism does not work. This formalism contains even auxiliary variables $\lambda^{\alpha}$ in addition to conventional odd variables $\theta^{\alpha}$. This idea is similar to the Pure Spinor construction. This paper is a short review of papers [11, 12]. Original results discussed below were obtained in collaboration with V. Alexandrov, A. Gorodentsev, A. Losev and V. Lysov.
\end{abstract}

\section{Introduction}

The central statement of the paper is that different classical field theories can be written in the form of action (2) by the addition of auxiliary fields. The fact that the theory (2) can be written in such a compact form is due to the introduction of the pure spinor fields $\mathcal{A}$ and $\mathcal{P}$ (the definition is given in Sect. 2). The construction of these fields was suggested in [1-3], [4]. What is claimed in this paper is that these fields can be considered as superfields and are needed to restore the off-shell SUSY invariance for the various on-shell supersymmetric theories. Another result is that the descent from off-shell supersymmetric action (2) down to its on-shell version is realized through the conventional procedure of quantum field theory - evaluation of the Feynman path integral. It happens that the perturbation series for this integral is finite, which means that it can be summed up explicitly.

As a universal framework for the calculation of "effective action" (elimination of auxiliary fields), we use the Batalin-Vilkovisky [6-10] formalism. This formalism allows us to control the descent of a symmetry of the action (2) down to its effective action. This technique is applied to the case of supersymmetry.

Another message of the present paper is that, in contrast to N. Berkovits' construction, the matrices $C_{\alpha \beta}^{\mu}$ in

a "New Talent" Presentation Award at the 44th Course of the International School of Subnuclear Physics, Erice,Italy, 29 Aug7 Sep 2006

e-mail: krotov@inr.ac.ru, krotov@itep.ru the definition of the pure spinor constraints (see Sect. 2 for details) are not necessarily Dirac $\gamma_{\alpha \beta}^{\mu}$-matrices. We consider them as arbitrary constant matrices. This "extension" allows us to obtain a plethora of different theories described by the construction.

In this short review we avoid rigorous proofs and technical details. Its aim is to explain our main ideas, presented in [11] and [12].

\section{A pure spinor superfield formalism}

The construction starts from the set of odd Grassmann variables $\theta^{\alpha}$ and an equal number of even variables $\lambda^{\alpha}$ (the index $\alpha$ runs in the range $\alpha=1 \ldots k$, where $k$ is arbitrary). These variables $\lambda^{\alpha}$ are restricted by the set of quadratic constraints $f^{\mu}=\lambda^{\alpha} C_{\alpha \beta}^{\mu} \lambda^{\beta}=0$. Here, $C_{\alpha \beta}^{\mu}$ are arbitrary constant matrices, symmetric in $\alpha, \beta$ (allowing only those combinations of $\lambda$ that are not proportional to $\left.f^{\mu}(\lambda)\right)$. In the end, the variables $\theta^{\alpha}$ will play the role of conventional supercoordinates. In a way that is similar to the "standard" approach, we introduce two superfields $\mathcal{A}$ and $\mathcal{P}$. $\mathcal{A}$ is a generic element in the space of $\theta^{\alpha}$ and $\lambda^{\alpha}$ with the component fields taking values in a (adjoint) representation of a given gauge group. The superfield $\mathcal{P}$ takes value in the space that is dual to that of $\theta^{\alpha}$ and $\lambda^{\alpha}$. The generating elements of this superfield are denoted by $\underline{\lambda}^{\alpha}$ and $\underline{\theta}^{\alpha}$. There is canonical pairing between the elements of the space of $\mathcal{A}$ and $\mathcal{P}$ denoted by $<,>$. It is defined as $<\underline{e}^{a}, e_{b}>=\delta_{b}^{a}$. Here, $e_{b}$ denotes basis elements in the space 
of $\mathcal{A}$ and $\underline{e}^{a}$ denotes basis elements in the space of $\mathcal{P}$. The number of basis elements $e_{b}$ is equal to the number of independent component fields in the superfield $\mathcal{A}$. The same is true for the superfield $\mathcal{P}$. The component fields in $\mathcal{A}$ and $\mathcal{P}$ are different. Introduction of the dual superfield is necessary to achieve the invariance of the construction under linear transformations of the basis elements $e_{a}$.

The next ingredient is the operator $[1-3,5]$

$$
Q_{B}=Q+\Phi=\lambda^{\alpha} \frac{\partial}{\partial \theta^{\alpha}}+\frac{1}{2} \theta^{\alpha} \frac{\partial f^{\mu}}{\partial \lambda^{\alpha}} \partial_{\mu} .
$$

Here $f^{\mu}(\lambda)$ is the set of quadrics defined above, and $\partial_{\mu}$ is the derivative with respect to the space-time coordinates. At this level we suppose that the number of quadrics is equal to the number of space-time coordinates. In Sect. 4 we will show (as described also in [11]) that one can make different reductions assuming that, for example, some derivatives $\partial_{\mu}$ act as zero on the component fields.

The central object of our consideration is the action

$$
S^{\text {Fund }}=\int \operatorname{Tr}\left(<\mathcal{P}, Q_{B} \mathcal{A}>+g<\mathcal{P}, \mathcal{A}^{2}>\right) .
$$

In this action, $\mathcal{A}$ and $\mathcal{P}$ are the superfields defined above and parameter $g$ is the gauge coupling constant. One can rewrite $S^{\text {Fund }}$ in terms of component fields. To find the contribution of the first term, one should apply the operator $Q_{B}$ to the generic superfield $\mathcal{A}$ and calculate the canonical pairing of the result with the superfield $\mathcal{P}$. In the second term, one should multiply the two $\mathcal{A}$ superfields, expand the result into the basis elements in the space of $\mathcal{A}$ and then evaluate the pairing $<,>$. The result of this calculation is some combination of component fields and space-time derivatives. Each component field is an element of a representation of the gauge group and depends on the space-time coordinates. To find the scalar action, one should calculate the trace over the gauge group indices and integrate over the space-time coordinates. These operations are presented in (2).

After the calculation of the canonical pairing $<,>$ the action (2) contains many fields. Some of these fields are physical and some of them are auxiliary. We would like to emphasize that the number of these fields and the way they contribute to the action is determined by the number $k$ of $\lambda^{\alpha}$ and $\theta^{\alpha}$ and the structure of the matrices $C_{\alpha \beta}^{\mu}$ in the definition of the quadrics. The crucial element of this construction is the assumption that among these fields the physical degrees of freedom are those which have a $\lambda-\theta$ structure that is proportional to the cohomologies of the operator $Q=\lambda^{\alpha} \frac{\partial}{\partial \theta^{\alpha}}$.

We would like to emphasize the difference between operators $Q_{B}$ and $Q$. The latter represents only the first term in $Q_{B}$. The physical spectrum of the theory coincides with the cohomologies of operator $Q$.

\section{Calculation of effective action}

Our strategy is to integrate out all the fields in the action (2) that are not cohomologies of the operator $Q$. The effective action on cohomologies is a classical BatalinVilkovisky action for a "physically interesting" theory. To avoid confusion, we would like to stress that the fields which are integrated out are auxiliary. Hence the terminology "effective action" may seem inappropriate for this object. However, as we will show below, the calculation of this "effective action" is the standard procedure in the BatalinVilkovisky formalism. In accordance with this observation, we retain this somewhat unconventional terminology.

\subsection{Batalin-Vilkovisky formalism}

In the approach to supersymmetric gauge theories that is the subject of the present paper, the Batalin-Vilkovisky formalism plays the role of universal framework for the calculation of effective action $[16,17]$. What is important for us is that the BV formalism is a convenient tool that allows us to control the descent of the arbitrary symmetry (global or local) of the "fundamental action" down to the effective action.

The central object in the formalism is the classical BV master equation

$$
0=\frac{\delta S^{\mathrm{BV}}}{\delta \chi^{n}} \frac{\delta S^{\mathrm{BV}}}{\delta \chi_{n}^{*}}
$$

Here $S^{\mathrm{BV}}$ is called BV action and depends on the fields, the ghosts and their BV antifields ( $\chi^{n}$ stands for all the fields of the theory, $\chi_{n}^{*}$ denotes the corresponding antifields). This equation has many solutions, but the most important one for us is called classical BV action

$$
S^{\mathrm{BV}}=S^{\mathrm{cl}}[A]+\delta A^{n} A_{n}^{*}+\frac{1}{2} f_{a b}^{c} c^{a} c^{b} c_{c}^{*}
$$

Here $S^{\mathrm{cl}}[A]$ is classical action, which depends only on the physical fields $A^{n}$ and is invariant under the symmetry transformations $\delta A^{n}$ of the second term. The parameter of these transformations is $c^{a}$. The fields $A_{n}^{*}$ and $c_{a}^{*}$ are BV antifields for $A^{n}$ and $c^{a}$. Parameters $f_{a b}^{c}$ are the structure constants for the algebra of symmetry. In the case of ordinary gauge symmetry, $\delta A^{n}$ is a BRST transformation of $A^{n}$ and $c^{a}$ is a Faddeev-Popov ghost.

It is important to note that if an action satisfies (3) and can be written in the form of classical BV action (4), this is equivalent to the following conditions:

- $S^{\mathrm{cl}}[A]$ is invariant under the algebra of symmetry of the second term.

- This algebra of symmetry is closed off-shell and the structure constants are given by $f_{a b}^{c}$.

The important point is that there exists a procedure for the calculation of the effective action that preserves the $\mathrm{BV}$ invariance. Namely, if the initial action satisfies the BV equation, then the effective action (which is obtained by integrating out some fields) satisfies the BV equation as well. The procedure involves integrating over a lagrangian submanifold in the space of fields $[16,17]$ (for the simple explanation see Sect. 2 of [11]). This fact makes it possible to analyse the symmetries of the effective action. 
We apply this technique to our calculation, starting from the action (2). It is possible to show that this action satisfies the BV equation (3). The BV fields are the component fields of $\mathcal{A}$, and the BV antifields are represented in the superfield $\mathcal{P}$. We are going to decompose the superfields $\mathcal{A}$ and $\mathcal{P}$ into two parts: $\mathcal{A}=A+a$ and $\mathcal{P}=P+p$. $A$ is the superfield that takes values in the space of $Q$ cohomologies, and $a$ lies in the orthogonal complement to the $Q$-cohomologies. $P$ and $p$ are the elements of spaces dual to those of $A$ and $a$. The effective action is defined as

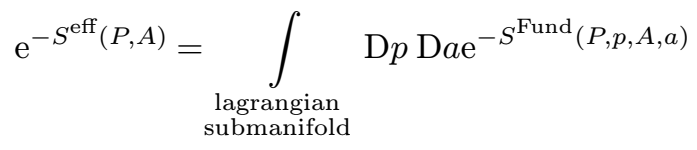

The fact that $S^{\text {Fund }}$ satisfies equation (3) is inherited in the gauge invariance of $S^{\text {eff }}(P, A)$.

Up to this point we have discussed the descent of the gauge invariance from the action (2) to $S^{\text {eff. }}$. This descent is "trivial" in the sense that both actions (2) and $S^{\text {eff }}$ are gauge invariant. In Sect. 5 we analyse the descent of supersymmetry from the same perspective.

\subsection{Feynman diagram technique}

In this subsection we explain how the calculation of $S^{\mathrm{eff}}(P, A)$ in (5) is done by summation of all possible connected Feynman diagrams, with external legs being the cohomologies of the $Q$-operator. In spite of the presence of the non-standard variables $\lambda$ and $\theta$ in the definition of $S^{\text {Fund }}$, this calculation is simply the calculation of a Feynman integral in conventional quantum field theory. The remarkable fact is that its perturbative expansion is finite and contains only a quite limited number of diagrams. These diagrams can be summed up explicitly into a rather simple and local effective action. Thus, the expansion of the integral (5) is exact.

Our suggestion is to consider the operator $\langle\mathcal{P}, Q \mathcal{A}\rangle$ (the first part of $Q_{B}$ ) as a kinetic term and the rest of $S^{\text {Fund }}$, namely $<\mathcal{P}, \Phi \mathcal{A}>$ and $g<\mathcal{P}, \mathcal{A}^{2}>$, as perturbation. With the decomposition $\mathcal{A}=A+a$ and $\mathcal{P}=P+p$, where $A$ and $P$ are defined by $Q$-cohomologies, it becomes apparent that the kinetic term reduces to $\langle p, Q a\rangle$. The terms $Q A$ and $P Q$ vanish. There are many contributions into the vertex part of the action. Some of them are $<P, \Phi A>,\left\langle p, \Phi A>,\left\langle P, \Phi a>,\langle p, \Phi a\rangle, g<P, a^{2}>\right.\right.$, ..., 12 contributions in total. Some of these vertices are drawn in Fig. 1 (only two vertices are illustrated here, but the remainder can be derived in an obvious way).

In this Figure, the solid in-coming line denotes the external field $A$, the solid out-going line denotes the field $P$, and the wavy lines denote the propagating fields $a$ and $p$.
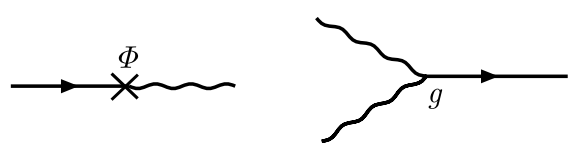

Fig. 1. Examples of vertices
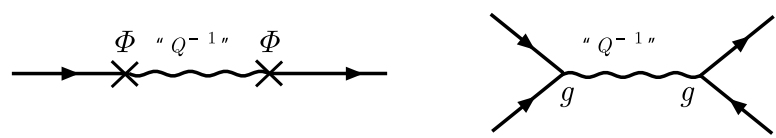

Fig. 2. Possible diagrams

We would like to emphasize that the kinetic term $<p, Q a>$ has a large number of zero modes (for example $\delta a=Q \varepsilon)$. To define the propagator " $Q{ }^{-1}$ " this gauge ambiguity should be fixed. This is done in (5) by restricting the domain of integration to the lagrangian submanifold.

Eventually, one could apply conventional Feynman technique to draw all possible diagrams. There are two rules to be followed.

- All quantum loops are absent in the theory (2).

- Even at the tree level, the length of the diagram is limited by $\theta$ structure. Indeed, propagator " $Q{ }^{-1}$ " increases the power of $\theta$. Since the number of $\theta^{\alpha}$ is equal to $k$, the number of propagators in each branch of the diagram can not be bigger than $k$. Usually this limit is even stronger, for instance, due to the $\lambda$ structure.

In Fig. 2 we present two possible diagrams as an example. The first one contributes to the kinetic term of the effective action, while the second one contributes to the interaction.

\section{The space of effective actions}

As already mentioned above, all information about degrees of freedom and the structure of effective action is encoded in the number $k$ of $\lambda$ and $\theta$ and the structure of quadrics $f^{\mu}(\lambda)$. It happens that there are two large classes of quadrics, which are called regular and singular systems. We do not give precise definitions [11] of these systems here. What is important for us is that for the case in which the number of quadrics is smaller than the number $k$, almost all possible systems are regular. In the bulk of regular systems there is a small but still infinite number of singular quadrics.

The point is that in the case of regular quadrics there is a certain universality in the spectrum of $Q$-cohomologies and hence in the structure of effective action. For an arbitrary regular system of quadrics, the effective theory is the AKSZ (Alexandrov, Kontsevich, Schwarz, Zaboronsky) version of the Chern-Simons theory (BF-theory) [13]. For the system of $N$ quadrics $f^{\mu}(\lambda)$ (index $\mu$ runs $\mu=$ $1 \ldots N)$ this theory is defined in $N$ dimensions. Therefore, in the regular case the effective action is not sensitive to the particular structure of matrices $C_{\alpha \beta}^{\mu}$ in the definition of quadrics. Only the number $N$ is important.

The opposite is true for singular systems. In this case the structure and degrees of freedom of effective action are highly sensitive to the structure of $C_{\alpha \beta}^{\mu}$. For each system of quadrics its own effective theory can be obtained. Though in the regular case effective action is a "trivial" topological theory, in the case of singular systems the effective action has a quite non-trivial spectrum and dynamics. Thus, we 


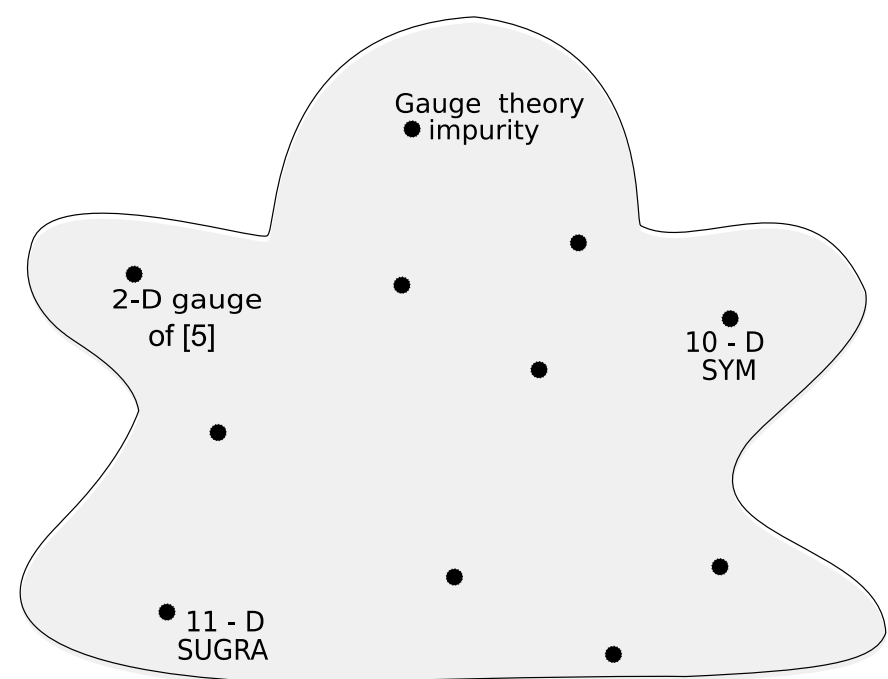

Fig. 3. The space of effective theories. The gray background represents the bulk of AKSZ theories. We emphasize that this picture is a schematic illustration only (see the comments below and footnote 1)

come to the conclusion that "physically interesting" theories are singularities in the bulk of trivial "topological" theories. This idea is schematically depicted in Fig. 3.

Until now, only a quite small number of singular systems has been investigated in detail. Some of them are depicted in the figure. It would be of great interest to search for such singular systems with different numbers $k$ of $\lambda$ and $\theta$ and different numbers $N$ and structures of quadrics.

It should be emphasized that this picture is only a schematic illustration. For example, the points " $2-d$ gauge model of [5]" and $10-d$ super Yang-Mills have different statuses. The action of $2-d$ gauge model is obtained from the fundamental action (2) exactly as outlined in the discussions of the previous Section. To obtain the action of $10-d \mathrm{SYM}$, on the other hand, an additional $Z_{2}$ projection must be made onto the space of fields (see Sect. 6). The same seems to be true for $11-d$ supergravity ${ }^{1}$.

We would also like to mention the possibility that there may be connections between these ideas and recent discussions regarding the Landscape program $[14,15]$.

\section{The descent of supersymmetry}

In the previous Sections we explained that by integrating out the orthogonal complement to $Q$-cohomologies in the action (2) one can obtain "physically interesting" effective action. In the present Section we show that all the fields that are integrated out are auxiliary. These fields are needed to restore the off-shell SUSY invariance for the onshell supersymmetric effective theory. The central statement is that the action $S^{\text {Fund }}$ is the off-shell supersymmetric version of $S^{\text {eff }}$ for a given system of quadrics. We also

1 To obtain the interaction terms for SUGRA, one should modify the interaction in the action (2), see [24]. show that for an arbitrary set of quadrics, the action $S^{\mathrm{eff}}$ is at least on-shell supersymmetric.

To prove these statements one uses the general ideology of BV formalism, as follows. To study the descent of supersymmetry from the action (2) to $S^{\text {eff }}$, one introduces the sources for each generator of the symmetry into the action. The algebra of SUSY is generated by $Q_{\alpha}^{s}$ and the translations $\partial_{\mu}$.

$$
Q_{\alpha}^{s}=\frac{\partial}{\partial \theta^{\alpha}}-C_{\alpha \beta}^{\mu} \theta^{\beta} \partial_{\mu} .
$$

The commutation relations are $\left\{Q_{\alpha}, Q_{\beta}\right\}=-2 C_{\alpha \beta}^{\mu} \partial_{\mu}$. This algebra is closed. We introduce the ghosts for SUSY $\varepsilon^{\alpha}$ and the ghosts for translations $\eta^{\mu}$. Then one can write the new action.

$$
S_{s}=S^{\text {Fund }}+\int \operatorname{Tr}\left(<\mathcal{P},\left(\varepsilon Q_{s}+\eta^{\mu} \partial_{\mu}\right) \mathcal{A}>-\eta_{\mu}^{*}\left(\varepsilon C^{\mu} \varepsilon\right)\right)
$$

Since we are discussing global symmetries, the ghosts $\varepsilon$ and $\eta$ do not depend on space-time coordinates. It is straightforward to check that the action (7) satisfies the BV equation (3) over the fields $\mathcal{A}, \mathcal{P}, \varepsilon^{\alpha}, \varepsilon_{\alpha}^{*}, \eta^{\mu}, \eta_{\mu}^{*}$ and has the structure of the classical BV action. Hence, according to the discussion in Sect. 3.1, the action $S^{\text {Fund }}$ is invariant under the SUSY transformations and the SUSY algebra is closed off-shell. Then we integrate out the complement to $Q$ cohomologies from the action (7) to obtain $S_{s}^{\text {eff. We do not }}$ integrate over parameters $\varepsilon^{\alpha}$ and $\eta^{\mu}$. Since the integration is done over a lagrangian submanifold, $S_{s}^{\text {eff }}$ also satisfies the classical BV equation. However, this action does not have the structure of (4). In addition to the standard BV structures, some "new" terms appear in $S_{s}^{\text {eff. }}$. These terms can be divided into two classes. We tried to determine the physical meaning of these terms [18-23]. Their definitions and interpretations are summarized in the Table 1 . Taking into account these facts, we conclude that if $S_{s}^{\text {eff }}$ satisfies the BV equation, then it follows that $S^{\text {eff }}$ (which is the first part of $S_{s}^{\text {eff }}$, independent of $\varepsilon$ and $\eta$ ) is on-shell supersymmetric.

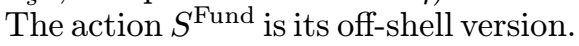

Table 1. Interpretation of non-standard terms in the master action

\begin{tabular}{ll}
\hline Term & Interpretation \\
\hline$\left(\varepsilon \psi^{*}\right)^{2}$ & $\begin{array}{l}\text { The terms are quadratic in antifields. The } \\
\text { appearance of such terms in BV action signals } \\
\text { that the algebra of symmetry is closed } \\
\text { only on-shell. }\end{array}$ \\
$c^{*}\left(\varepsilon \gamma^{\mu} \varepsilon\right) A_{\mu} \quad \begin{array}{l}\text { Such terms are responsible for the gauge } \\
\text { fixing. For example, they appear in the } \\
\text { BV action when one fixes the Wess-Zumino } \\
\text { gauge in } \mathcal{N}=1, D=4 \text { SYM theory. The } \\
\text { appearance of such terms signals that the } \\
\text { algebra of SUSY is closed only up to some } \\
\text { gauge transformation. }\end{array}$ \\
\hline
\end{tabular}


As a concluding remark, we would like to emphasize that the introduction of ghosts $\varepsilon$ and $\eta$ is only a technical trick, which allows us to control the SUSY properties of $S^{\text {Fund }}$ and $S^{\text {eff }}$. On the other hand, the fields $\mathcal{A}$ and $\mathcal{P}$ can be considered as superfields. All this is true for the theories, similar to [11], which do not require $Z_{2}$ projection. For the $Z_{2}$ projected theories the situation is a little bit different.

\section{The descent of SUSY in $Z_{2}$ projected theories}

We start this Section with the simplest example of $Z_{2}$ projected theories - 10-dimensional SYM. This system is described [1-3] by the set of quadrics $f^{\mu}(\lambda)=\lambda^{\alpha} \gamma_{\alpha \beta}^{\mu} \lambda^{\beta}$, where $\gamma_{\alpha \beta}^{\mu}$ are 10 dimensional Dirac $\gamma$-matrices (index $\alpha$ runs in the range $\alpha=1 \ldots 16$, index $\mu$ runs over $\mu=1 \ldots 10$ ). The spectrum of cohomologies is given in the Table 2 .

The first column presents all the fields of effective theory, and the second column contains their BV antifields. From this table one can see that in addition to the fields $\left(c, A_{\mu}, \psi^{\alpha}\right)$ (which are $\mathrm{F}-\mathrm{P}$ ghost, gauge field and gluino) and their antifields $\left(c^{*}, A_{\mu}^{*}, \psi_{\alpha}^{*}\right)$, which are needed for the $\mathrm{BV}$ action of SYM, there are additional fields with the tilde sign. These additional fields are not simply decoupled from effective action, but actually contribute to it in a rather non-trivial way. To obtain the action of SYM, one should carry out $Z_{2}$ reduction, by factorizing out the tilde-fields according to the rule $\widetilde{c}^{*}=-c, \widetilde{A}_{\mu}^{*}=A_{\mu}, \widetilde{\psi}^{*}=-\psi, \widetilde{\psi}=$ $\psi^{*}, \widetilde{A}_{\mu}=-A_{\mu}^{*}, \widetilde{c}=c^{*}$. After this $Z_{2}$ reduction, the effective action calculated for (2) reduces to BV action for SYM.

We would like to emphasize that although the calculation of effective action before $Z_{2}$ projection for the theory (2) is a well-defined procedure in quantum field theory, $Z_{2}$ projection itself is done "by hand". We do not yet know how it can be realized through the Feynman path integral. A quite similar situation holds for the case of 11- $d$ supergravity. It would be interesting to understand how this

Table 2. The spectrum of cohomologies

\begin{tabular}{lcc}
\hline Polarization & $A$ & $P$ \\
\hline 1 & $\mathrm{c}$ & $c^{*}$ \\
$\left(\lambda \gamma^{\mu} \theta\right)$ & $A_{\mu}$ & $A_{\mu}^{*}$ \\
$\left(\lambda \gamma^{\mu} \theta\right)\left(\theta \gamma_{\mu}\right)_{\alpha}$ & $\psi^{\alpha}$ & $\psi^{* \alpha}$ \\
$\left(\lambda \gamma^{\mu} \theta\right)\left(\lambda \gamma^{\nu} \theta\right)\left(\theta \gamma_{\mu \nu}\right)^{\alpha}$ & $\widetilde{\psi}_{\alpha}$ & $\widetilde{\psi}_{\alpha}^{*}$ \\
$\left(\lambda \gamma^{\mu} \theta\right)\left(\lambda \gamma^{\nu} \theta\right)\left(\theta \gamma_{\mu \nu \rho} \theta\right)$ & $\widetilde{A}_{\rho}$ & $\widetilde{A}_{\rho}^{*}$ \\
$\left(\lambda \gamma^{\mu} \theta\right)\left(\lambda \gamma^{\nu} \theta\right)\left(\lambda \gamma^{\rho} \theta\right)\left(\theta \gamma_{\mu \nu \rho} \theta\right)$ & $\widetilde{c}$ & $\widetilde{c}^{*}$ \\
\hline
\end{tabular}

$Z_{2}$ projection can be realized within the framework of BV formalism (evaluation of path integral). This would shed light on the structure of off-shell SUSY invariant actions for these $Z_{2}$ projected theories (including 10-d SYM, 11-d SUGRA, etc.).

Acknowledgements. It is a pleasure to thank the organizers of the International School of Subnuclear Physics (Erice, 2006) for suggesting the idea to write this review, and for the excellent atmosphere of the School. I am very indebted to V. Alexandrov, A. Losev, V. Lysov and V. Rubakov for useful critical discussions. This work was supported by the grant RFBR-0502-17363- a and the grant of the President of the Russian Federation NS-7293.2006.2 (government contract 02.445.11.7370) and Dynasty foundation in 2007.

\section{References}

1. N. Berkovits, JHEP 0109, 016 (2001) [hep-th/0105050]

2. N. Berkovits, JHEP 0004, 018 (2000) [hep-th/0001035]

3. N. Berkovits, hep-th/0209059

4. B.E.W. Nilsson, Class. Quantum Grav. 3, L41 (1986)

5. E. Witten, Nucl. Phys. B 266, 245 (1986)

6. I.A. Batalin, G.A. Vilkovisky, Phys. Lett. B 102, 27 (1981)

7. I.A. Batalin, G.A. Vilkovisky, Phys. Rev. D 28, 2567 (1983)

8. I.A. Batalin, Phys. Rev. D 30, 508 (1984)

9. B.L. Voronov, I.V. Tyutin, Theor. Math. Phys. 50, 218 (1982)

10. B.L. Voronov, Teor. Mat. Fiz. 50, 333 (1982)

11. D. Krotov, A. Losev, [hep-th/0603201]

12. V. Alexandrov, D. Krotov, A. Losev, V. Lysov, arXiv: hep-th/0705.2191

13. M. Alexandrov, M. Kontsevich, A. Schwartz, O. Zaboronsky, Int. J. Mod. Phys. A 12, 1405 (1997) [hep-th/9502010]

14. L. Susskind, hep-th/0302219

15. J. Polchinski, hep-th/0603249

16. A. Schwarz, Commun. Math. Phys. 155, 249 (1993) [hep-th/9205088]

17. A. Schwarz, Commun. Math. Phys. 158, 373 (1993) [hep-th/9210115]

18. E.S. Fradkin, M.A. Vasiliev, Phys. Lett. B 72, 70 (1977)

19. M.B. Green, C.M. Hull, QMC/PH/89-7. Presented at Texas A and M Mtg. on String Theory, College Station, TX, Mar 13-18, 1989

20. M.B. Green, C.M. Hull, Nucl. Phys. B 344, 115 (1990)

21. M.B. Green, C.M. Hull, Mod. Phys. Lett. A 5, 1399 (1990)

22. E. Bergshoeff, R. Kallosh, A. Van Proeyen, Class. Quantum Grav. 9, 321 (1992)

23. R.E. Kallosh, Phys. Lett. B 225, 49 (1989)

24. L. Anguelova, P.A. Grassi, P. Vanhove, Nucl. Phys. B 702, 269 (2004) [hep-th/0408171] 